\title{
EFEITOS DE INSETICIDAS, FUNGICIDAS E BIORREGULADORES NA QUALIDADE FISIOLÓGICA DE SEMENTES DE SOJA DURANTE O ARMAZENAMENTO
}

\author{
Andreia da Silva Almeida ${ }^{1}$, César Iván Suárez Castellanos ${ }^{1}$, Cristiane Deuner ${ }^{1}$, Carolina Terra \\ Borges $^{1}$, Geri Eduardo Meneghello ${ }^{1}$
}

${ }^{1}$ Universidade Federal de Pelotas, E-mail: carol_tborges@hotmail.com

\section{RESUMO}

O objetivo deste trabalho foi testar misturas de fungicidas, inseticidas e biorreguladores, no tratamento de sementes, sobre a qualidade fisiológica de sementes de soja. O tratamento de sementes com quaisquer das misturas testadas contribuiu para que a perda de vigor das sementes, causada pelo armazenamento, fosse mais lenta quando comparada com sementes sem tratamento.

Palavras-chave: Soja, tratamento de sementes, vigor de sementes, geminação

\section{EFFECT OF INSECTICIDES, FUNGICIDES AND BIOREGULATORS IN THE PHYSIOLOGICALQUALITY OF SOYBEAN SEEDS DURING STORAGE}

\begin{abstract}
The aim of this research was to test, via seed treatment, the effect of mixtures of fungicides, insecticides and bioregulators in the physiological quality of soybean seeds. Seed treatment with any mixture tested in this study does contribute for the loss of seed vigor, caused by storage, to be slower when compared to non treated seeds.
\end{abstract}

Keywords: Soybean, seed treatment, seed vigor, germination

\section{INTRODUÇÃO}

A soja é a principal oleaginosa cultivada no mundo e sua produção se destaca no cenário nacional, ocupando nas últimas décadas, aproximadamente $50 \%$ da área agrícola plantada (CONAB, 2013).

Vários avanços na tecnologia da produção de soja têm ocorrido para que a área e o rendimento da cultura tenham aumentado nos últimos anos e dentre desses encontra-se a qualidade das sementes usadas nas lavouras. Segundo Baudet \& Peske (2007) e Silva (1998), a germinação uniforme das sementes e o crescimento vigoroso e homogêneo das plântulas no início do desenvolvimento da cultura são fatores essenciais para garantir o máximo 
potencial produtivo contido geneticamente nas mesmas. Igualmente, Baudet \& Peres (2004) observaram que sementes com alto poder germinativo, emergência a campo e potencial de crescimento podem produzir um estande inicial uniforme que será refletido numa alta produtividade da cultura. No entanto, uma vez semeadas no campo as sementes ficam expostas a vários fatores bióticos (pragas e doenças) e abióticos que podem afetar seu desempenho genético e fisiológico, diminuindo sua germinação e alterando a uniformidade de emergência das plântulas. Por essa razão e com o objetivo de proteger as sementes e mudas na fase inicial do crescimento contra todo tipo de adversidades, produtos fitossanitários como fungicidas e inseticidas são aplicados nas sementes (LUDWIG et al., 2011; PEREIRA et al., 2011).

Segundo Avelar et al. (2011) o tratamento de sementes de soja é uma técnica economicamente recomendada, desde que utilizados produtos ou misturas de produtos adequados, na dosagem recomendada e distribuídos uniformemente em todo o lote de sementes. Da mesma forma, Lucca Filho (2006) determinou que um tratamento químico eficiente deve selecionar um produto capaz de erradicar os patógenos presentes nas sementes, não ser tóxico às plantas, ao homem e ao ambiente, apresentar alta estabilidade, aderência e cobertura, não ser corrosivo, ser de baixo custo e fácil aquisição, além de ser compatível com outros produtos.

Conforme aumenta a percepção do valor da semente e a importância de proteger e/ou melhorar o seu desempenho, cresce no mercado a disponibilidade de produtos para o tratamento de sementes, com diferentes finalidades, como proteção (fungicidas ou inseticidas) ou nutrição (micronutrientes), tendo como finalidade melhorar $\mathrm{O}$ desempenho da semente, tanto no aspecto fisiológico como econômico (AVELAR et al., 2011).

Vários trabalhos tem sido feitos com a mistura de inseticidas e fungicidas com outros produtos, dentre os quais se encontram micronutrientes, bioativadores, biorreguladores, bioestimulantes e polímeros (BAYS et al., 2007; ÁVILA et al., 2008; AVELAR et al., 2011; LUDWIG et al., 2011; DOURADO-NETO et al., 2012).

Os biorreguladores são compostos orgânicos, não nutrientes que, aplicados na planta, a baixas concentrações, promovem, inibem ou modificam processos morfológicos e fisiológicos do vegetal, por exemplo, as auxinas. Os bioestimulantes são misturas de um ou mais biorreguladores com 
outros compostos de natureza química diferente, como sais minerais (CASTRO \& PEREIRA, 2008). Os bioativadores são substâncias naturais de origem vegetal que possuem ações semelhantes aos principais reguladores vegetais, que podem favorecer o crescimento e o desenvolvimento da planta (CASTRO et al., 2008). Já os polímeros utilizados no tratamento de sementes proporcionam aumento do tamanho e peso das sementes, alteração do formato, melhoria da eficiência e da distribuição dos produtos sobre a semente, proteção do operador contra a contaminação com produtos químicos, redução da variação de temperatura e umidade e agregação de valor comercial às sementes. Conforme Reichenbach (2004) os polímeros podem aumentar a retenção e uniformidade de distribuição de fungicidas na superfície das sementes.

Contudo, além de aumentar a proteção das sementes e auxiliar no desenvolvimento inicial das plântulas, os produtos usados no tratamento de sementes e suas misturas não devem interferir de forma negativa sobre a qualidade fisiológica dos lotes de sementes, seja imediatamente após o tratamento ou após o armazenamento.

Considerando as ideias descritas anteriormente, o objetivo do presente trabalho foi testar várias misturas de produtos com propriedades fungicidas, inseticidas e bioestimulantes, via tratamento de sementes, sobre a qualidade fisiológica das mesmas através do tempo.

\section{MATERIAL E MÉTODOS}

O experimento foi realizado no Laboratório Didático de Análise de Sementes- LDAS do programa de pósgraduação em Ciência e Tecnologia de Sementes da Universidade Federal de Pelotas, localizado no município de Capão do Leão - RS.

Sementes de soja da cultivar NK7059RR, foram tratadas usando tratadora Niklas com rotação de $25 \mathrm{~Hz}$ e ciclo de aplicação de 15 minutos (5 min de injeção, 8 min misturando, $2 \mathrm{~min}$ descarga), constituindo 7 tratamentos (Tabela 1).

Após o tratamento, as sementes foram secas em ar ambiente, colocadas em sacos de papel e armazenadas em câmara fria (umidade relativa de $40 \%$ e temperatura de $16^{\circ} \mathrm{C}$ ) até a condução das análises. Foram realizados testes de germinação, envelhecimento acelerado e emergência a campo em três momentos, logo após o tratamento, 90 dias após o tratamento e 180 dias após o tratamento. 
Tabela 1. Produtos e doses utilizadas no tratamento de sementes de soja, cultivar NK7059RR, Capão do Leão-RS, 2014.

\begin{tabular}{|c|c|c|c|c|}
\hline Tratamento & $\begin{array}{c}\text { Produto } \\
\text { Comercial }\end{array}$ & Ingrediente ativo & Concentração & Dose utilizada \\
\hline $\mathrm{T} 1$ & - & - & - & $\begin{array}{c}\text { Testemunha } \\
\text { (Sem tratamento) }\end{array}$ \\
\hline \multirow{3}{*}{$\mathrm{T} 2$} & Crusier 350FS & Thiamethoxam & $350 \mathrm{~g} \mathrm{~L}^{-1}$ & $2,5 \mathrm{~mL} \mathrm{Kg} \mathrm{sementes}^{-1}$ \\
\hline & Maxim Advanced & Fludioxonil & $25 \mathrm{~d} \mathrm{~L}^{-1}$ & $1 \mathrm{~mL} \mathrm{Kg} \mathrm{sementes}{ }^{-1}$ \\
\hline & FloRite 1197 & Polímero & - & $0,5 \mathrm{~mL} \mathrm{Kg}$ sementes $^{-1}$ \\
\hline \multirow{4}{*}{$\mathrm{T} 3$} & Crusier 350FS & Thiamethoxam & $350 \mathrm{~g} \mathrm{~L}^{-1}$ & $2 \mathrm{~mL} \mathrm{Kg} \mathrm{sementes}{ }^{-1}$ \\
\hline & Maxim Advanced & Fludioxonil & $25 \mathrm{~d} \mathrm{~L}^{-1}$ & $1 \mathrm{~mL}$ Kg sementes ${ }^{-1}$ \\
\hline & Avicta 500FS & Abamectina & $500 \mathrm{~g} \mathrm{~L}^{-1}$ & $1 \mathrm{~mL} \mathrm{Kg}$ sementes $^{-1}$ \\
\hline & FloRite 1197 & Polímero & - & $0,5 \mathrm{~mL} \mathrm{Kg}$ sementes $^{-1}$ \\
\hline \multirow{6}{*}{$\mathrm{T} 4$} & Crusier 350FS & Thiamethoxam & $350 \mathrm{~g} \mathrm{~L}^{-1}$ & $2 \mathrm{~mL} \mathrm{Kg}$ sementes $^{-1}$ \\
\hline & Maxim Advanced & Fludioxonil & $25 \mathrm{~d} \mathrm{~L}^{-1}$ & $1 \mathrm{~mL}$ Kg sementes ${ }^{-1}$ \\
\hline & & Cinetina & $0,09 \mathrm{~g} \mathrm{~L}^{-1}$ & \multirow{3}{*}{$5 \mathrm{~mL}$ Kg sementes ${ }^{-1}$} \\
\hline & Stimulate & $\mathrm{GA}_{3}$ & $0,05 \mathrm{~g} \mathrm{~L}^{-1}$ & \\
\hline & & $\begin{array}{l}\text { Ácido 4-indol-3- } \\
\text { ilbutírico }\end{array}$ & $0,05 \mathrm{~g} \mathrm{~L}^{-1}$ & \\
\hline & FloRite 1197 & Polímero & - & $0,5 \mathrm{~mL} \mathrm{Kg}$ sementes $^{-1}$ \\
\hline \multirow{7}{*}{ T5 } & Crusier 350FS & Thiamethoxam & $350 \mathrm{~g} \mathrm{~L}^{-1}$ & $2 \mathrm{~mL} \mathrm{Kg} \mathrm{sementes}{ }^{-1}$ \\
\hline & Maxim Advanced & Fludioxonil & $25 \mathrm{~d} \mathrm{~L}^{-1}$ & $1 \mathrm{~mL} \mathrm{Kg} \mathrm{\text {sementes } ^ { - 1 }}$ \\
\hline & Avicta 500FS & Abamectina & $500 \mathrm{~g} \mathrm{~L}^{-1}$ & $1 \mathrm{~mL} \mathrm{Kg} \mathrm{sementes}{ }^{-1}$ \\
\hline & & Cinetina & $0,09 \mathrm{~g} \mathrm{~L}^{-1}$ & \multirow{3}{*}{$5 \mathrm{~mL} \mathrm{Kg} \mathrm{sementes}{ }^{-1}$} \\
\hline & Stimulate & GA3 & $0,05 \mathrm{~g} \mathrm{~L}^{-1}$ & \\
\hline & & $\begin{array}{l}\text { Ácido 4-indol-3- } \\
\text { ilbutírico }\end{array}$ & $0,05 \mathrm{~g} \mathrm{~L}^{-1}$ & \\
\hline & FloRite 1197 & Polímero & - & $0,5 \mathrm{~mL} \mathrm{Kg} \mathrm{sementes}^{-1}$ \\
\hline \multirow{4}{*}{ T6 } & Crusier 350FS & Thiamethoxam & $350 \mathrm{~g} \mathrm{~L}^{-1}$ & $2 \mathrm{~mL} \mathrm{Kg} \mathrm{sementes}{ }^{-1}$ \\
\hline & Maxim Advanced & Fludioxonil & $25 \mathrm{~d} \mathrm{~L}^{-1}$ & $1 \mathrm{~mL}$ Kg sementes ${ }^{-1}$ \\
\hline & Booster Agrichem & Co e Mo & NI & 2,5 mL Kg sementes ${ }^{-1}$ \\
\hline & FloRite 1197 & Polímero & - & $0,5 \mathrm{~mL} \mathrm{Kg}$ sementes $^{-1}$ \\
\hline \multirow{7}{*}{$\mathrm{T} 7$} & Crusier 350FS & Thiamethoxam & $350 \mathrm{~g} \mathrm{~L}^{-1}$ & $2 \mathrm{~mL} \mathrm{Kg} \mathrm{sementes}{ }^{-1}$ \\
\hline & Maxim Advanced & Fludioxonil & $25 \mathrm{~d} \mathrm{~L}^{-1}$ & $1 \mathrm{~mL} \mathrm{Kg}$ sementes $^{-1}$ \\
\hline & & Cinetina & $0,09 \mathrm{~g} \mathrm{~L}^{-1}$ & \multirow{3}{*}{$5 \mathrm{~mL} \mathrm{Kg} \mathrm{sementes}{ }^{-1}$} \\
\hline & Stimulate & GA3 & $0,05 \mathrm{~g} \mathrm{~L}^{-1}$ & \\
\hline & stiminate & $\begin{array}{l}\text { Ácido 4-indol-3- } \\
\text { ilbutírico }\end{array}$ & $0,05 \mathrm{~g} \mathrm{~L}^{-1}$ & \\
\hline & Booster Agrichem & Co e Mo & NI & 2,5 mL Kg sementes ${ }^{-1}$ \\
\hline & FloRite 1197 & Polímero & - & $0,5 \mathrm{~mL} \mathrm{Kg} \mathrm{sementes}^{-1}$ \\
\hline
\end{tabular}


Teste de germinação: Realizado através da semeadura de 200 sementes por repetição, distribuídas em quatro rolos de 50 sementes cada, sobre papel germitest ${ }^{\circledR}$ umedecido com água destilada em proporção $2,5 \mathrm{p} / \mathrm{v}$. Após a montagem dos rolos as sementes foram transferidas para germinador, regulado à temperatura constante de $25^{\circ} \mathrm{C}$. A contagem foi realizada aos cinco e oito dias após a semeadura, conforme as Regras para Analise de Sementes - RAS (BRASIL, 2009). Os resultados foram expressos em porcentagem de plântulas normais.

Teste de envelhecimento acelerado: Realizado através da colocação de uma camada de sementes sobre uma tela metálica acoplada a uma caixa plástica tipo gerbox contendo $40 \mathrm{~mL}$ de água destilada no fundo (mini-câmara). As caixas foram tampadas e acomodadas em câmara tipo BOD por 48 horas a $41^{\circ} \mathrm{C}$ (MARCOS FILHO, 2005). Após este período, as sementes foram colocadas para germinar conforme as RAS e aos cinco dias foi feita a contagem de plântulas normais, expressando os resultados em porcentagem.

Teste de emergência a campo: Realizado conforme o procedimento sugerido por Nakagawa (1999), semeando-se 50 sementes por repetição. A avaliação foi realizada em contagem única aos 21 dias depois da semeadura, determinando o número de plântulas normais e expressando os resultados em porcentagem. $\mathrm{O}$ modelo estatístico usado foi de delineamento inteiramente casualizado em esquema de parcelas divididas com quatro repetições onde o fator parcela foi o tempo. Os resultados obtidos foram submetidos à análise de variância e as médias dos tratamentos comparadas pelo teste de Tukey a $5 \%$ de probabilidade, usando o programa estatístico WinStat 1.0 (MACHADO \& CONCEIÇÃO, 2003). Antes da análise, os dados foram transformados usando o método do $\operatorname{arc} \cdot \operatorname{sen} \sqrt{\left(\frac{x}{100}\right)}$.

\section{RESULTADOS E DISCUSSÃO}

Conforme a análise de variância observou-se que houve interação entre o tempo e o tratamento para as variáveis germinação e envelhecimento acelerado. Já a emergência a campo mostrou resposta ao tempo e aos tratamentos, porém não a interação entre eles.

Observa-se que a porcentagem de germinação das sementes no tempo zero de avaliação foi estatisticamente igual em todos os tratamentos. Já nos 90 e 180 dias após tratamento, observou-se que não houve 
diferença significativa entre os tratamentos 3 a 7, sendo esses os que mostraram uma germinação maior. $\mathrm{O}$ tratamento 2 (Crusier 350 FS + Maxim Advanced + FloRite 1197) teve germinação maior que a testemunha porém menor que os demais tratamentos. Tavares et al. (2007) não observaram diferenças na germinação de sementes de soja tratadas com várias doses de thiamethoxam. De forma semelhante, Dan et al. (2011) observaram que sementes de soja tratadas com thiamethoxam, fipronil e imidacloprid não apresentaram diferenças na germinação quando comparadas com a testemunha. Pereira et al. (2009) observaram que o tratamento de sementes de soja com diferentes fungicidas, incluindo 0 fludioxonil, não interferiram sobre a germinação e emergência das sementes em bandeja. Igualmente se observou que a germinação diminuiu do tempo zero para o tempo de 90 dias e se manteve até os 180 dias em todos os tratamentos, com exceção do tratamento 4 (Crusier $350 \mathrm{FS}+$ Maxim Advanced + Stimulate + FloRite 1197), o qual não apresentou diferenças significativas entre as três épocas avaliadas neste trabalho (Tabela 2).
Dan et al. (2010) avaliando a qualidade fisiológica de sementes de soja tratadas com os inseticidas thiamethoxam, fipronil, imidacloprid, imidacloprid + thiodicarb, carbofuran e acefato sob efeito do armazenamento verificaram uma redução da porcentagem de germinação das sementes conforme aumentou o período de armazenamento, após o tratamento com os inseticidas, com destaque para o acefato e o carbofuran. Segundo Antonello et al. (2009), os princípios ativos podem afetar o desenvolvimento de plântulas sob condições adversas, imediatamente após o tratamento, ou após um curto período de armazenamento. Em contrapartida, Pires et al. (2004), verificaram que o tratamento de sementes com fungicidas e polímeros não prejudicou a germinação de sementes de feijoeiro durante quatro meses de armazenamento. Kunkur et al. (2007) observaram que o tratamento de sementes de algodão com fungicida, inseticida e polímero propiciou sementes com maior porcentagem de germinação após nove meses de armazenamento. 
Tabela 2. Germinação (\%), envelhecimento acelerado (\%) e emergência a campo (\%) de sementes de soja, cultivar NK7059RR tratadas com vários produtos, Capão do LeãoRS, 2014.

\begin{tabular}{|c|c|c|c|c|c|c|}
\hline \multirow{2}{*}{ Variável Resposta } & \multirow{2}{*}{ Tratamento } & \multicolumn{3}{|c|}{ Tempo (dias após tratamento) } & \multirow{2}{*}{ Média } & \multirow{2}{*}{$\mathrm{CV} \%$} \\
\hline & & 0 & 90 & 180 & & \\
\hline \multirow{8}{*}{ Germinação (\%) } & $\mathrm{T} 1$ & $96 \mathrm{aA}$ & $78 \mathrm{~b} \mathrm{C}$ & $78 \mathrm{~b} \mathrm{C}$ & 84 & \multirow{8}{*}{2,42} \\
\hline & $\mathrm{T} 2$ & $98 \mathrm{aA}$ & $85 \mathrm{~b} \mathrm{~B}$ & $83 \mathrm{~b} \mathrm{~B}$ & 89 & \\
\hline & T3 & $98 \mathrm{aA}$ & $91 \mathrm{~b} \mathrm{~A}$ & $89 \mathrm{~b} \mathrm{~A}$ & 93 & \\
\hline & $\mathrm{T} 4$ & $94 \mathrm{aA}$ & $91 \mathrm{aA}$ & $90 \mathrm{aA}$ & 92 & \\
\hline & T5 & $96 \mathrm{aA}$ & $91 \mathrm{~b} \mathrm{~A}$ & $89 \mathrm{~b} \mathrm{~A}$ & 92 & \\
\hline & T6 & $94 \mathrm{aA}$ & $90 \mathrm{~b} \mathrm{~A}$ & $89 \mathrm{~b} \mathrm{~A}$ & 91 & \\
\hline & $\mathrm{T} 7$ & $95 \mathrm{aA}$ & $91 \mathrm{~b} \mathrm{~A}$ & $89 \mathrm{~b} \mathrm{~A}$ & 92 & \\
\hline & Média & 96 & 88 & 87 & - & \\
\hline \multirow{8}{*}{ Envelhecimento Acelerado (\%) } & $\mathrm{T} 1$ & $95 \mathrm{aA}$ & $80 \mathrm{~b} \mathrm{~B}$ & $77 \mathrm{~b} \mathrm{C}$ & 84 & \multirow{8}{*}{2,38} \\
\hline & $\mathrm{T} 2$ & $96 \mathrm{aA}$ & $90 \mathrm{~b} \mathrm{~A}$ & $82 \mathrm{c} \mathrm{BC}$ & 89 & \\
\hline & $\mathrm{T} 3$ & $94 \mathrm{aA}$ & 89 b A & $87 \mathrm{~b} \mathrm{~A}$ & 90 & \\
\hline & $\mathrm{T} 4$ & $93 \mathrm{aA}$ & $89 \mathrm{~b} \mathrm{~A}$ & $87 \mathrm{~b} \mathrm{~A}$ & 90 & \\
\hline & T5 & $94 \mathrm{aA}$ & $87 \mathrm{~b} \mathrm{~A}$ & $86 \mathrm{~b} A B$ & 89 & \\
\hline & T6 & $94 \mathrm{aA}$ & $88 \mathrm{~b} \mathrm{~A}$ & $85 \mathrm{~b} \mathrm{AB}$ & 89 & \\
\hline & $\mathrm{T} 7$ & $94 \mathrm{aA}$ & $89 \mathrm{~b} \mathrm{~A}$ & $86 \mathrm{~b} A B$ & 90 & \\
\hline & Média & 94 & 87 & 84 & - & \\
\hline \multirow{8}{*}{ Emergência a Campo (\%) } & T1 & 94 & 90 & 81 & $88 \mathrm{~B}$ & \multirow{8}{*}{2,17} \\
\hline & $\mathrm{T} 2$ & 97 & 98 & 87 & $94 \mathrm{~A}$ & \\
\hline & T3 & 97 & 95 & 88 & $93 \mathrm{~A}$ & \\
\hline & $\mathrm{T} 4$ & 97 & 97 & 89 & $94 \mathrm{~A}$ & \\
\hline & $\mathrm{T} 5$ & 97 & 97 & 88 & $94 \mathrm{~A}$ & \\
\hline & T6 & 97 & 97 & 86 & $93 \mathrm{~A}$ & \\
\hline & $\mathrm{T} 7$ & 97 & 98 & 87 & $94 \mathrm{~A}$ & \\
\hline & Média & $96 \mathrm{a}$ & $96 \mathrm{a}$ & $86 \mathrm{~b}$ & - & \\
\hline
\end{tabular}

* Médias seguidas pela mesma letra, minúscula na linha e maiúscula na coluna, não diferem entre si pelo teste de

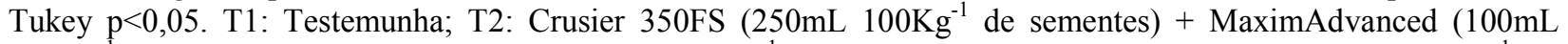
$100 \mathrm{Kg}^{-1}$ de sementes $)+$ FloRite $1197\left(50 \mathrm{~mL} 100 \mathrm{Kg}^{-1}\right.$ de sementes $)$; T3: Crusier $350 \mathrm{FS}\left(200 \mathrm{~mL}^{100 \mathrm{Kg}^{-1}} \mathrm{de}\right.$

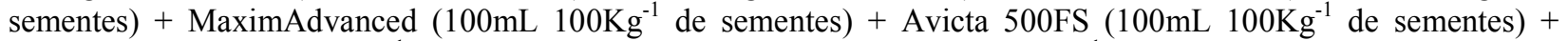
FloRite $1197\left(50 \mathrm{~mL}^{100 \mathrm{Kg}^{-1}}\right.$ de sementes); T4: Crusier 350FS (200mL $100 \mathrm{Kg}^{-1}$ de sementes) + MaximAdvanced $\left(100 \mathrm{~mL}^{100 \mathrm{Kg}^{-1}}\right.$ de sementes $)+$ Stimulate $\left(500 \mathrm{~mL} 100 \mathrm{Kg}^{-1}\right.$ de sementes $)+$ FloRite $1197\left(50 \mathrm{~mL}^{100 \mathrm{Kg}^{-1}} \mathrm{de}\right.$

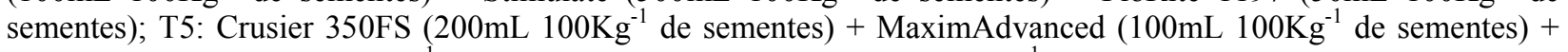
Avicta 500FS $\left(100 \mathrm{~mL}^{100 \mathrm{Kg}^{-1}}\right.$ de sementes) + Stimulate $\left(500 \mathrm{~mL}^{100 \mathrm{Kg}^{-1}}\right.$ de sementes $)+$ FloRite $1197(50 \mathrm{~mL}$ $100 \mathrm{Kg}^{-1}$ de sementes); T6: Crusier 350FS (200mL $100 \mathrm{Kg}^{-1}$ de sementes) + MaximAdvanced $\left(100 \mathrm{~mL}^{100 \mathrm{Kg}^{-1}} \mathrm{de}\right.$ sementes) + BoosterAgrichem $\left(250 \mathrm{~mL} 100 \mathrm{Kg}^{-1}\right.$ de sementes $)$ + FloRite $1197\left(50 \mathrm{~mL}^{100 \mathrm{Kg}^{-1}}\right.$ de sementes) e T7: Crusier 350FS (200mL $100 \mathrm{Kg}^{-1}$ de sementes) + MaximAdvanced $\left(100 \mathrm{~mL}^{100 \mathrm{Kg}^{-1}}\right.$ de sementes $)+$ Stimulate

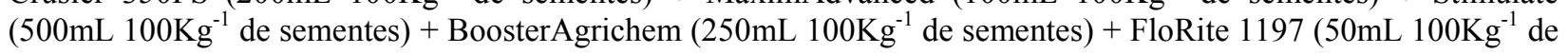
sementes). 
O vigor das sementes, mensurado através do teste de envelhecimento acelerado, foi estatisticamente igual para todos os tratamentos no tempo zero de avaliação. Aos 90 dias após tratamento se observou que a testemunha foi significativamente menor do que os demais tratamentos. Já na avaliação aos 180 dias, os tratamentos 3 (Crusier 350FS + Maxim Advanced + Avicta 500FS + FloRite 1197) e 4 (Crusier 350FS + Maxim Advanced + Stimulate + FloRite 1197) mostraram maior porcentagem de plântulas normais do que os demais, enquanto a testemunha e o tratamento 2 (Crusier 350FS + Maxim Advanced + FloRite 1197) apresentaram o menor número de plântulas normais.Também se observou que o vigor das sementes foi significativamente maior na época zero de avaliação quando comparada com as outras duas (90 e 180 dias) em todos os tratamentos. No entanto, em todos os tratamentos, excetuando o T2, não houve diferença significativa entre as épocas 90 e 180 dias após tratamento. No tratamento 2 (Crusier 350FS + Maxim Advanced + FloRite 1197) o vigor das sementes foi diminuindo significativamente através do tempo (Tabela 2). Piccinin et al. (2013) verificaram que o tratamento de sementes com os inseticidas fipronil e thiamethoxam prejudicou a qualidade fisiológica das sementes quando submetidas ao armazenamento por 180 dias em condições normais de armazenamento.

A emergência a campo foi menor na testemunha do que nos outros tratamentos, não tendo diferenças significativas entre os tratamentos 2 a 7. Grisi et al. (2009) também não constataram alteração no vigor e na emergência das sementes de girassol tratadas com thiamethoxam e fipronil. Em relação às épocas de avaliação pode-se constatar que não houve diferenças significativas entre as épocas 0 e 90 dias, as quais mostraram uma porcentagem de emergência significativamente maior do que a emergência a campo avaliada aos 180 dias após o tratamento de sementes (Tabela 2). Dan et al. (2010) afirmaram que é possível ocorrer efeito fitotóxico, em decorrência do aumento do período de armazenamento das sementes tratadas com inseticidas. Guerra et al. (2006) concluíram que o Co e o Mo, aplicados via tratamento de sementes, incrementaram a germinação e emergência a campo das sementes da geração subsequente na cultura da soja. Por outra parte, Moraes et al. (2001) constataram que sementes tratadas com carboxin + thiram 180 dias antes da semeadura, não tiveram alteração da qualidade fisiológica. 


\section{CONCLUSÕES}

O tratamento de sementes com a mistura de Crusier 350FS + Maxim Advanced + Stimulate + FloRite 1197 mantém a germinação de sementes de soja por até 180 dias.

O tratamento de sementes com qualquer mistura testada neste trabalho contribui para que a perda de vigor das sementes causada pelo armazenamento seja mais lenta quando comparada com sementes sem tratamento.

O tratamento de sementes com os produtos testados aumenta e mantém a emergência a campo das sementes por até 90 dias.

\section{REFERÊNCIAS BIBLIOGRÁFICAS}

ANTONELLO, L.M.; MUNIZ, M.B.; BRAND, S.C.; VIDA, M.D.; GARCIA, D.; RIBEIRO, L.; SANTOS, V.2009. Qualidade de sementes de milho armazenadas em diferentes embalagens. Ciência Rural, Santa Maria, v.39, n.7, p. 2191-2194.

AVELAR, S.A.G.; BAUDET, L.; PESKE, S.T.; LUDWIG, M.P.; RIGO, G.A.; CRIZEL, R.L.; OLIVEIRA, S. 2011. Storage of soybean seed treated with fungicide, insecticide and micronutrient and coated with liquid and powered polymer. Ciência Rural, Santa Maria,v.41, n.10, p.1719-1725.

ÁVILA, M.R.; BRACCINI, A.L.; SCAPIM, C.A.; ALBRECHT, L.P.; TONIN, T.A.; STÜLP, M. 2008. Bioregulator application, agronomic efficiency, and quality of soybean seeds. Scientia Agricola, Piracicaba, v.65, n.6, p.604612.

BARROS, R.G.; YOKOYAMA, M.; COSTA, J.L. da S. 2001. Compatibilidade do inseticida thiamethoxan com fungicidas utilizados no tratamento de sementes de feijoeiro. Pesquisa Agropecuária Tropical, Goiás, v.31, n.2, p.153-157.

BAUDET, L.; PERES, W.B. 2004. Recobrimento de sementes. Seed News, Pelotas, v.4, n. 1, p. 20-23.

BAUDET, L.; PESKE, F. 2007. Aumentando o desempenho das sementes. Seed News, v.9, n.5, p.22-24.

BAYS, R.; BAUDET, L.; HENNING, A.A.; LUCCA FILHO, O. 2007. Recobrimento de sementes de soja com micronutrientes, fungicida e polímero. Revista Brasileira de Sementes, Londrina, v.29, n.2, p.60-67.

BRASIL. Ministério da Agricultura, Pecuária e Abastecimento. 2009.

Regras para análise de sementes. Brasília: Mapa/ACS.

CASTRO, P.R.C.; PEREIRA, M.A. Bioativadores na agricultura. In: GAZZONI, D.L. (Coord.).2008. Tiametoxam: uma revolução na agricultura brasileira. Petrópolis: Vozes, p. 115-122.

CASTRO, G.S.A.; BOGIANI, J.C.; SILVA, M.G.; GAZOLA, E.; ROSOLEM, C.A. 2008. Tratamento de sementes de soja com inseticidas e um bioestimulante. Pesquisa Agropecuária Brasileira, Brasília, DF, v. 43, n. 10, p. 1311-1318.

COMPANHIA NACIONAL DE ABASTECIMENTO (CONAB). 2013. Acompanhamento de safra brasileira: grãos. Brasília, DF: Conab.

DAN, L.G.M.; DAN, H.A.; BARROSO, A. L.L.; BRACCINI, A. L. 2010. Qualidade fisiológica de sementes de soja tratadas com inseticidas sob efeito 
do armazenamento. Revista Brasileira de Sementes, Londrina, v. 32, n. 2 p. 131-139.

DAN，L.G.M.; DAN, H.A.; BRACCINI, A.L.; ALBRECHT, L.P.; RICCI, T.T.; PICCININ, G.G. 2011. Desempenho de sementes de soja tratadas com inseticidas e submetidas a diferentes períodos de armazenamento. Revista Brasileira de Ciências Agrárias, Recife, v.6. n. 2, p.215-222.

DOURADO-NETO, D.; DARIO, G.J.A.; MARTIN, T.N.; SILVA, M. R.; PAVINATO, P.S.; HABITZREITER, T.L. 2012. Adubação mineral com cobalto e molibdênio na cultura da soja.

Semina: Ciências Agrárias, Londrina, v.33, p.2741-2752.

GRISI, P.U.; SANTOS, C.M.; FERNANDES, J.J.; SÁ JÚNIOR, A. 2009. Qualidade das sementes de girassol tratadas com inseticidas $\mathrm{e}$ fungicidas. Bioscience Journal, Uberlândia, v.25, n.4, p.28-36.

GUERRA， C.A.; MARCHETTI， M.E.; ROBAINA, A.D.; SOUZA, L.C.F.; GONÇALVES, M.C.; NOVELINO, J.O. 2006. Qualidade fisiológica de sementes de soja em função da adubação com fósforo, molibdênio e cobalto. Acta Scientiarum Agronomy, Maringá, v. 28. n.1. p.91-97.

KUNKUR, V.; HUNJE, R.; PATIL, N.K.B.; VYAKARNHAL, B.S. 2007. Effect of Seed Coating with Polymer, Fungicide and Insecticide on Seed Quality in Cotton During Storage. Karnataka Journal of Agricultural Sciences, Dharwad, v.20, n.1, p.137139.http://203.129.218.157/ojs/index.ph $\mathrm{p} / \mathrm{kjas} /$ article/viewFile/42/42.

LUCCA FILHO, O.A. Patologia de Sementes. In.: PESKE, S.T.; LUCCA FILHO, O.A.; BARROS, A.C.S.A. (Ed.). 2006. Sementes: fundamentos científicos e Tecnológicos, 2.Ed., Pelotas, p.259-329.
LUDWIG, M.P.; LUCCA FILHO, O.A.; BAUDET, L.; DUTRA, L.M.C.; AVELAR, S.A.G.; CRIZEL, R.L. 2011. Qualidade de sementes de soja armazenadas após recobrimento com aminoácido, polímero, fungicida e inseticida. Revista Brasileira de Sementes, Londrina, v.33, n.3, p.395406.

MARCOS FILHO, J. 2005. Fisiologia de sementes de plantas cultivadas. Piracicaba: FEALQ, $495 \mathrm{p}$.

MORAES, M.H.D.; MARUOKA, A.E.; GRAVENA, C.; MENTEN, J.O.M.; DIAS, P.M.F. 2001. Avaliação da eficiência do tratamento antecipado de sementes de soja com carboxin + thiram. Fitopatologia Brasileira, Brasília, v.26, p.426.

NAKAGAWA, J. Testes de vigor baseados no desempenho das plântulas. In: KRZYZANOWSKI, F. C.; VIEIRA, R. D.; FRANÇA-NETO, J. B. 1999. Vigor de sementes: conceitos e testes. Londrina: ABRATES, Cap. 2, p. 9-13.

PEREIRA， C.E.; OLIVEIRA， J.A.; OLIVEIRA, G.E.; ROSA, M.C.M.; NETO, J.C. 2009. Tratamento fungicida via peliculização e inoculação de Bradyrhizobium em sementes de soja. Revista de Ciências Agronômicas, v. 40, n.3, p.433-440.

PEREIRA, C.E.; GUIMARÃES, R.M.; VIEIRA, A.R.; EVANGELISTA, J.R.E.; OLIVEIRA， G.E. 2011. Tratamento fungicida e peliculização de sementes de soja submetidas ao armazenamento. Ciência Agrotécnica, Lavras, v.35, n.1, p.158-164.

PICCININ, G.G.; BRACCINI, A.L.; DAN, L.G. de M.; BAZO, G.L.; LIMA, L.H. da S. 2013. Influência do armazenamento na qualidade fisiológica de sementes de soja tratadas com inseticidas. Ambiência, Guarapuava, v.9 n. 2 p. $289-298$. 
PIRES, L.L.; BRAGANTINI, C.; COSTA, J.L.S.2004. Armazenamento de sementes de feijão revestidas com polímeros e tratadas com fungicidas. Pesquisa agropecuária brasileira, Brasília, D.F., v.39, n.7, p.709-715.

REICHENBACH, J. 2004. Film-coating para agregar qualidade e segurança. Seed News, Pelotas, n.1. p.24-25

SILVA, M.T.B. 1998. Inseticidas na proteção de sementes e plantas. Seed News, Pelotas, v.2, n.5, p.26-27.

TAVARES, S.; CASTRO, P.R.C.; RIBEIRO, R.V.; ARAMAKI, P.H. 2007. Avaliação dos efeitos fisiológicos de thiametoxan no tratamento de sementes de soja. Revista de Agricultura, Piracicaba, v.82, n.1, p.4754. 